\title{
Independent particle model for nucleon transfer in heavy ion collisions
}

\author{
C. Gnucci and Fl. Stancu \\ Institut de Physique, B5, Université de Liège, Sart Tilman, B-4000 Liège 1, Belgium \\ L. Wilets \\ Physics Department, University of Washington, Seattle, Washington 98195
}

(Received 3 October 1983)

\begin{abstract}
We formulate a three-dimensional quantum-mechanical model for the study of nucleon transfer in heavy ion collisions. This model describes the independent particle motion of nucleons initially separated in two adjacent cubic boxes and brought into contact through a window created in the separating wall. We calculate the one-sided flux and the spread of the mass distribution and compare them with results derived from other models.
\end{abstract}

\section{INTRODUCTION}

The particle exchange mechanism plays an important role in explaining the dissipative phenomena observed in heavy ion collisions. ${ }^{1}$ Within the one-body dissipation mechanism ${ }^{2}$ the relevant quantity for the dissipation rate is the one-sided current of nucleons which are exchanged via a window ${ }^{3}$ opened between the interacting nuclei.

Recently attempts have been made to derive the onesided current on a microscopic basis, starting either from time-dependent ${ }^{4}$ or adiabatic time-dependent ${ }^{5}$ HartreeFock wave functions. In such calculations some care must be taken because the quantum-mechanical definition $^{6,7}$ of the one-sided current does not always have a meaning. ${ }^{8}$ This is due to the fact that the Wigner transform of the one-body density may assume regions of negative values which prevent the extension of the classical definition of the one-sided current ${ }^{2,3}$ to a quantum system.

In the present work we formulate a simple threedimensional quantum-mechanical model in order to obtain more insight into the dynamics of particle exchange. This model describes the independent particle motion of nucleons initially separated in two adjacent cubic boxes and brought into contact through a window created in the separating wall. We calculate a time-dependent one-sided current in two different ways. One is according to the currently used definition ${ }^{4-8}$ where the flux is evaluated as in Eq. (3.1) from the Wigner distribution function $f(\vec{r}, \vec{k}, t)$ of the one-body density. The other is given by the initial conditions of our model and better visualizes the physics contained in it. We start at $t=0$ with one box empty and the other full. At $t>0$ the window (suddenly) opens, the wave functions spread out of the filled box, and the matter flows into the empty box. As the box has a finite size, the waves travel up to the opposite wall where they are reflected. We study the correspondence between this matter flow and the result obtained from the currently used definition of the one-sided current. We also make a comparison between the presently obtained time averaged and the static one-sided current calculated in Ref. 9, at the same time indicating the value of the purely geometrical flux.
As we deal with an entirely quantum-mechanical system which simulates the mechanism of particle exchange we can also calculate the fluctuations $\sigma^{2}$ in the number of particles. The result is compared to that obtained by Buck and Feldmeier ${ }^{7}$ in a one-dimensional model without two-body collisions. The above authors indicated that such a term has a negligible effect on the order of magnitude of $\sigma^{2}$.

In Sec. II we give a brief account of the static model and introduce the time-dependent wave functions. In Sec. III we present results for the matter flow calculated according to various definitions. Section IV deals with the calculation of the width of the mass distribution, and Sec. $\mathrm{V}$ is devoted to the conclusion.

\section{THE MODEL}

Our model consists of two adjacent hard-walled cubes having a length $L$ on each edge and communicating through a window initially closed. Up to time $t=0$ the boxes are equally filled with $A / 2$ particles each. At $t=0$ the window opens and the particles move freely from one box to the other through the window. The geometry is indicated in Fig. 1. The window is situated in the plane $z=0$ and extends from $x=-w$ to $x=w$ and from $y=-L / 2$ to $y=L / 2$. The Hamiltonian is symmetric under reflections through each coordinate. We denote by $\pi_{i}$ the parity for $i=x, y$, or $z$ and choose the basis functions

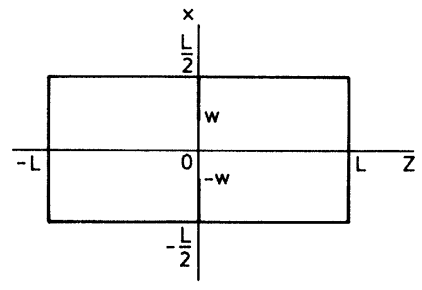

FIG. 1. Adjacent cubic boxes communicating through a rectangular window of size $x=2 w, y=L$ in the $z=0$ plane. 


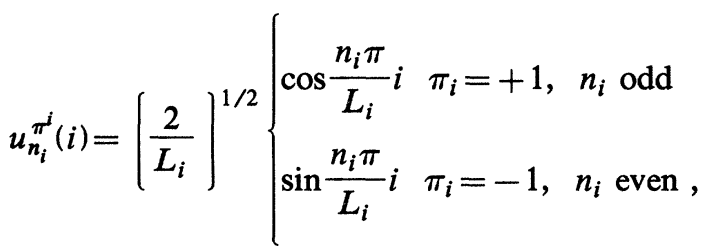

which vanish on the planes $L_{i} / 2$, where $L_{x}=L_{y}=L$ and $L_{z}=2 L$.

The system is described by functions defined inside the volume occupied by the two cubes. They have total parity $\pi=\pi_{x} \pi_{y} \pi_{z}$ and due to the geometry of the window they can be factorized as

$$
\phi_{\alpha, n_{y}}^{\pi}(\vec{r})=u_{n_{y}}^{\pi_{y}}(y) \phi_{\alpha}^{p}(x, z)
$$

with $p=\pi_{x} \pi_{z}$ and $\phi_{\alpha}^{p}$ given by the expansion

$$
\phi_{\alpha}^{p}(x, z)=\sum_{n} c_{\alpha n}^{p} u_{n_{x}}^{\pi_{x}}(x) u_{n_{z}}^{\pi_{z}}(z)
$$

where the summation runs through $n=\left(n_{x}, n_{z}\right)$ and the coefficients $c_{\alpha n}^{p}$ are determined from the boundary conditions imposed on the separating wall which requires the vanishing of the wave function at $z=0, w \leq|x| \leq L / 2$. Such a condition is automatically satisfied for $\pi_{z}=-1$ and achieved only approximately for $\pi_{z}=+1$ states. The procedure has been described in detail in Ref. 9 and it is equivalent with solving the Schrödinger equation for the following potential:

$$
V=\lambda \delta(z) \theta(|x|-w) ; \lambda \rightarrow \infty,
$$

in a truncated space, i.e., solving the matrix equation

$$
\sum_{n^{\prime}}\left[\left(\epsilon_{n}^{p}-E_{\alpha}^{p}\right) \delta_{n n^{\prime}}+V_{n^{\prime}}^{p}\right] c_{\alpha n^{\prime}}^{p}=0,
$$

where

$$
\epsilon_{n}^{p}=\frac{\hbar^{2}}{2 m} \frac{\pi^{2}}{L^{2}}\left(n_{x}^{2}+\frac{1}{4} n_{z}^{2}\right)
$$

and

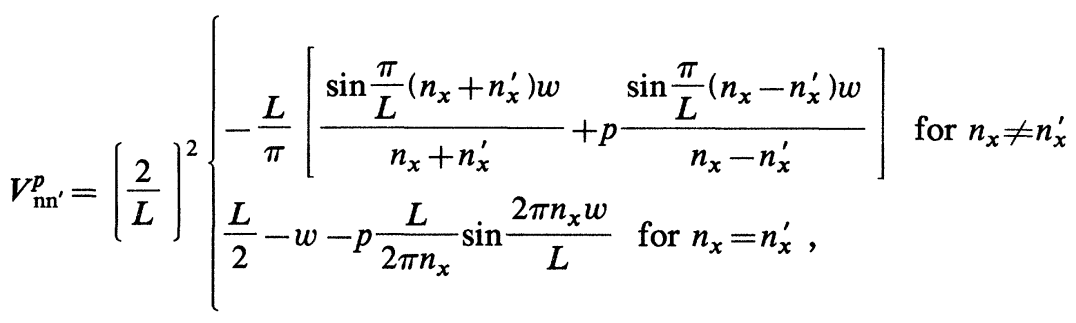

for any $n_{z}$ and $n_{z}^{\prime}$ odd, or equivalently $\pi_{z}=+1$, or else we have

$$
V_{\mathrm{nn}^{\prime}}^{p}=0 \text { for } \pi_{z}=-1
$$

as automatically implied by (2.1) and (2.4). Now let us call $N$ the maximum value taken by $n_{z}$ in the truncated space where the matrix equation (2.5) is solved. The case $N+1$ even reduces to $N$ odd due to (2.8). At fixed $N$ odd and for $\pi_{z}=1, n_{x}$ runs up to $N$ for $p=+1$ and up to $N-1$ for $p=-1$. Hence the model has two parameters, $N$ and $\lambda$. Adequate values of these parameters have been found in Ref. 9. Accordingly, in the present calculations we choose $\lambda=10^{4} \mathrm{MeV}$ fm and $N=19$ whenever $w<L / 2$.

Now, when the procedure of obtaining the eigenstates (2.2) of the communicating boxes has been settled, we can introduce the time-dependent wave functions. At $t=0$ we assume that the window is closed and the particles fill one of the boxes, let us say for example the left box. For states initially localized in the left box $(z<0)$ we shall use the label $L$. Then at $t>0$ the associated single particle wave function $\psi_{n}^{L}$ will evolve in time according to

$$
\psi_{n}^{L}(x, z, t)=\sum_{\alpha} d_{n \alpha}^{\pi_{x} p}(L) e^{-(i / \hbar) E_{\alpha}^{p_{t}}} \phi_{\alpha}^{p}(x, z) .
$$

The variable $y$ is omitted for simplicity but taken into account in filling up the levels (see Table I). The states $\psi_{n}^{R}$ initially localized in the right box and orthogonal to $\psi_{n}^{L}$ are defined in a similar way with the associated expansion coefficients $d_{n \alpha}^{\pi_{x} p}(R)$. In both cases the coefficients are en- tirely determined by the initial conditions as the overlap

\begin{tabular}{|c|c|c|c|c|c|}
\hline System & $n_{x}$ & $n_{y}$ & $n_{z}$ & $\begin{array}{c}\epsilon_{n}^{\pi} \\
(\mathrm{MeV})\end{array}$ & $\begin{array}{l}\text { Occupation } \\
\text { number } \\
\text { Eq. }(2.15)\end{array}$ \\
\hline \multirow{4}{*}{$A=32$} & 1 & 1 & 2 & 29.69 & 0.9998 \\
\hline & 1 & 2 & 2 & 59.40 & 0.9998 \\
\hline & 2 & 1 & 2 & 59.40 & 0.9998 \\
\hline & 1 & 1 & 4 & 59.40 & 0.9994 \\
\hline \multirow{10}{*}{$A=80$} & 1 & 1 & 2 & 16.11 & 0.9998 \\
\hline & 1 & 2 & 2 & 32.23 & 0.9998 \\
\hline & 2 & 1 & 2 & 32.23 & 0.9998 \\
\hline & 1 & 1 & 4 & 32.23 & 0.9994 \\
\hline & 2 & 2 & 2 & 48.35 & 0.9998 \\
\hline & 2 & 1 & 4 & 48.35 & 0.9994 \\
\hline & 1 & 2 & 4 & 48.35 & 0.9994 \\
\hline & 1 & 1 & 6 & 59.10 & 0.9985 \\
\hline & 3 & 1 & 2 & 59.10 & 0.9998 \\
\hline & 1 & 3 & 2 & 59.10 & 0.9998 \\
\hline
\end{tabular}
integrals

$d_{n \alpha}^{\pi_{x} p}(L)=\int u_{n}^{L} \phi_{\alpha}^{p} d v$ and $d_{n \alpha}^{\pi_{x} p}(R)=\int u_{n}^{R} \phi_{\alpha}^{p} d v$,

TABLE I. The occupied states in a cubic box at $t=0$ (closed window) for $A=32$ and 80 particle systems. The energy eigenvalue is

$$
\epsilon_{n}^{\pi}=\hbar^{2} / 2 m\left(\frac{\pi}{L}\right)^{2}\left(n_{x}^{2}+n_{y}^{2}+\frac{1}{4} n_{z}^{2}\right)
$$


where

$$
u_{n}^{L}=\frac{2}{L} \theta(-z) \sin \frac{\pi n_{z}}{2 L} z\left\{\begin{array}{l}
\sin \frac{\pi n_{x}}{L} x ; n_{x} \text { even, } n_{z} \text { even } \\
\cos \frac{\pi n_{x}}{L} x ; n_{x} \text { odd, } n_{z} \text { even }
\end{array}\right.
$$

and

$$
u_{n}^{R}(z)=u_{n}^{L}(-z) ; z>0 .
$$

We are reminded that $p$ is the parity associated with the eigenstate $\phi_{\alpha}^{p}$ under reflections in the $x, z$ plane and $\pi_{x}$ is the parity of the state (2.11) at reflection through the $x$ axis. The product $\pi_{x} p= \pm 1$ serves as the upper index for the coefficients (2.10) and indicates that the function (2.9) has a given parity at reflections through the $x$ axis but mixes symmetric and antisymmetric states $\phi_{\alpha}^{p}$ under reflections through the $z$ axis.

The integrals (2.10) give

$$
\begin{aligned}
& d_{n \alpha}^{-1}(L)=d_{n \alpha}^{-1}(R)=\frac{\sqrt{2}}{2} \delta_{n \alpha}, \\
& d_{n \alpha}^{+1}(L)=-d_{n \alpha}^{+1}(R)=\frac{\sqrt{2}}{L} \sum_{n^{\prime}} c_{\alpha n^{\prime}}^{p} N_{n_{z} n_{z}^{\prime}} \delta_{n_{x} n_{x}^{\prime}},
\end{aligned}
$$

with

$$
N_{n_{z} n_{z}^{\prime}}=-\frac{L}{\pi}\left(\frac{1}{n_{z}+n_{z}^{\prime}}+\frac{1}{n_{z}-n_{z}^{\prime}}\right) ; n_{z} \text { even, } n_{z}^{\prime} \text { odd . }
$$

In the present work we consider two systems, $A=32$ and 80 particles, respectively. The occupied states at $t=0$ when the window is closed and each box contains $A / 2$ particles are indicated in Table I for both systems, each state being four times degenerate. They are given in increasing order of the energy

$$
\frac{\hbar^{2}}{2 m}\left[\frac{\pi}{L}\right]^{2}\left(n_{x}^{2}+n_{y}^{2}+\frac{1}{4} n_{z}^{2}\right)
$$

We note that $n_{z}$ is always even, indicating the cancellation of the wave function at $z=0$ (closed window). The size $L$ is chosen such as to have an average density $\rho=0.17 \mathrm{fm}^{-3}$ in each box. This gives $L=4.55$ and $6.17 \mathrm{fm}$, for $A=32$ and 80 particles, respectively. Now we want to approximate the eigenstates of the left (or right) box by the truncated expansion (2.9). As an example in Fig. 2 we plot $\psi_{n}^{L}$ at $t=0, x=y=0$ as a function of $z ; n_{x}$ can take any odd value and the four curves correspond to $n_{z}=2,4,6$, and 8 , respectively. The truncation has been made at $N=15$, i.e., the summation in Eq. (2.9) contains one state with $p=-1$ $\left(\alpha_{z}\right.$ even) and eight states with $p=+1\left[\alpha_{z}\right.$ (odd) $\left.\leq N\right]$. We notice that the wave function $\psi_{n}^{L}$ is very well localized. We found that a larger space, having $N>15$, brings insignificant changes to the wave functions with $n_{z} \leq 8$ when $w=L / 2$ and therefore limits the expansion at $N=15$ for a fully open window. A quantitative measure of the localization is given by the probability of finding the particle

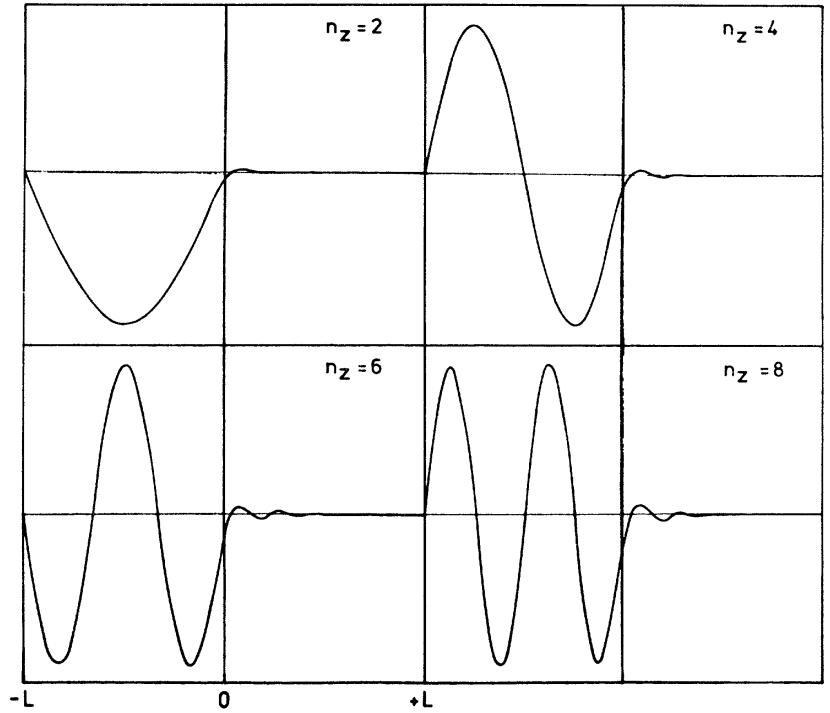

FIG. 2. Eigenstates of the left box $L / \sqrt{2} \psi_{n}^{L}$ at $t=0, x=0$. They are given by the truncated expansion (2.9) containing nine eigenstates of the big box (fully open window), i.e., $N=15$. The value of $n_{z}$ is specified for each case; $n_{x}$ can take any odd value.

within the volume of the left box

$$
\int_{L} d v\left|\psi_{n}^{L}(t=0)\right|^{2}=\sum_{\alpha}\left[d_{n \alpha}^{\pi_{x} p}(L)\right]^{2} .
$$

This is indicated in the last column of Table I for all initially occupied states. For $n_{z}=8$ states, unoccupied at $t=0$, it is about 0.997 , i.e., close to 1 in all cases of interest. The latter will be used in Sec. IV for calculating the width of the mass distribution.

A comment is necessary about the truncation for the case $w<L / 2$. A first truncation is made in search for the state $\phi_{\alpha}^{p}$. Not all these states are physically acceptable. The vanishing of the wave function on the intermediate wall is achieved to a very good approximation for the lowest states. The associated eigenvalue $E_{\alpha}^{p}$ becomes practically independent of $\lambda$. The highest excited states do not have such properties. But, in order to localize the wave function in the left or right box, it turned out that two or three of the lowest states carry most of the weight for all occupied and few unoccupied states close to the Fermi level. Hence the truncated space used in the preceding section to define $\phi_{\alpha}^{p}$ can be even more restricted in (2.9). A practical alternative is to keep the same dimension, but instead of $E_{\alpha}^{p}$ in (2.9), we use the expectation values $\left\langle\phi_{\alpha}^{p}|T| \phi_{\alpha}^{p}\right\rangle$ of the kinetic energy operator which permits us to get rid of the region where $E_{\alpha}^{p}$ depends strongly on $\lambda$ for the highest excited states. For all the states where the vanishing of the wave function on the intermediate wall is well achieved $E_{\alpha}^{p}$ and the expectation value of the kinetic energy are practically the same. By using $\left\langle\phi_{\alpha}^{p}|T| \phi_{\alpha}^{p}\right\rangle$ instead of $E_{\alpha}^{p}$ one can also ensure the satisfaction of the continuity equation. Details are discussed in Ref. 10.

\section{MASS EXCHANGE}

A measure of the number of nucleons exchanged through the window is the one-sided flux. ${ }^{2,3}$ In this sec- 
tion we calculate the one-sided flux by using two different definitions and discuss the relation between the two calculations. We also compare the present results with the static one-sided flux. ${ }^{9}$

\section{A. Definition I}

This is an extension of the definition used in classical mechanics and it makes use of the Wigner distribution function $f(\overrightarrow{\mathrm{r}}, \overrightarrow{\mathrm{k}}, t)$ associated with the one-body density of the quantum system. According to this definition the one-sided flux in the $z$ direction is given by di, $^{4,8,9}$

$$
j_{+}(\overrightarrow{\mathrm{r}}, t)=\frac{\hbar}{m} \int_{k_{z} \geq 0} d^{3} k k_{z} f(\overrightarrow{\mathrm{r}}, \overrightarrow{\mathrm{k}}, t) .
$$

This also represents the expectation value of the quantum-mechanical operator introduced by Feldmeier. ${ }^{6,7}$ Classically the distribution function is positive, hence $j_{+}$ is also positive, but for quantum systems problems might arise because the Wigner function can have negative values. An example has been extensively discussed in Ref. 8.

Using definition (3.1), we calculate the one-sided current through the window, i.e., at $z=0$,

$$
J_{+}(t)=\int_{-w}^{w} d x \int_{-L / 2}^{L / 2} d y j_{+}(x, y, 0, t)
$$

in the following way. At $t=0$ the window is closed and each box contains $A / 2$ particles. The occupied states $\psi_{n}^{L}$ $(t=0)$ of the left box are degenerate with $\psi_{n}^{R}(t=0)$ of the right box. Adding together the contribution of each two initially degenerate states we define the one-sided flux associated with the state $n$,

$$
j_{+, n}(\overrightarrow{\mathrm{r}}, t)=j_{+, n}^{L}(\overrightarrow{\mathrm{r}}, t)+j_{+, n}^{R}(\overrightarrow{\mathrm{r}}, t) .
$$

Then, the total flux reads

$$
j_{+}(\overrightarrow{\mathrm{r}}, t)=4 \sum_{n(\mathrm{occ})} j_{+, n}(\overrightarrow{\mathrm{r}}, t)
$$

when the sum runs over all occupied states, listed in Table $I$ and the factor 4 takes into account the spin-isospin degeneracy. Here by $n$ we mean the set $n_{x}, n_{y}, n_{z}$. In filling up the levels in increasing order it is necessary for each state to add the contribution $\left(\hbar^{2} / 2 m\right)(\pi / L)^{2} n_{y}^{2}$ to $E_{\alpha}^{p}$. We are reminded that the $y$-dependent part of the wave function appearing as the factor $u_{n_{y}}^{\pi_{y}}(y)$ remains invariant in the process of building up the wave function $\psi_{n}^{L}$ or $\psi_{n}^{R}$ and was therefore omitted for simplicity.

From the Wigner function,

$$
f_{n}(\overrightarrow{\mathrm{r}}, \overrightarrow{\mathrm{k}}, t)=\frac{1}{(2 \pi)^{3}} \int d^{3} s e^{-i \overrightarrow{\mathrm{k}} \cdot \overrightarrow{\mathrm{s}}} \psi_{n}^{*}\left[\overrightarrow{\mathrm{r}}+\frac{\overrightarrow{\mathrm{s}}}{2}\right] \psi_{n}\left[\overrightarrow{\mathrm{r}}-\frac{\overrightarrow{\mathrm{s}}}{2}\right],
$$

of each occupied state we calculate $j_{+, n}^{L}$ and $j_{+, n}^{R}$ according to definition (3.1), and making the operations (3.2)-(3.4), we obtain

$$
J_{+}(t)=\frac{\hbar}{m} \frac{1}{L^{2}} \sum_{n(\mathrm{occ})} \sum_{\alpha, \alpha^{\prime}} d_{n \alpha}^{\pi_{x} p}(L) d_{n \alpha^{\prime}}^{\pi_{x} p}(L) F_{\alpha \alpha^{\prime}}^{p} e^{(i / \hbar)\left(E_{\alpha}^{p}-E_{\alpha^{\prime}}^{p}\right) t},
$$

with

$$
F_{\alpha^{\prime} \alpha^{\prime \prime}}^{p}=\sum_{n^{\prime}, n^{\prime \prime}} c_{\alpha^{\prime} n^{\prime}}^{p} c_{\alpha^{\prime \prime} n^{\prime \prime}}^{p} B_{n_{x}^{\prime} n_{x}^{\prime \prime}}^{\pi_{x}}\left\{\left(n_{z}^{\prime}-n_{z}^{\prime \prime}\right) \operatorname{Si}\left[\left(n_{z}^{\prime}-n_{z}^{\prime \prime}\right) \frac{\pi}{2}\right]+\left(n_{z}^{\prime}+n_{z}^{\prime \prime}\right) \operatorname{Si}\left[\left(n_{z}^{\prime}+n_{z}^{\prime \prime}\right) \frac{\pi}{2}\right]\right\},
$$

where $\operatorname{Si}(x)$ is the sine integral ${ }^{11}$ and

$$
B_{n_{x}^{\prime} n_{x}^{\prime \prime}}^{\pi_{x}}=\delta_{n_{x}^{\prime} n_{x}^{\prime \prime}}-\frac{L}{2 \lambda} V_{n_{x}^{\prime} n_{x}^{\prime \prime}}^{\pi_{x}}
$$

with $V_{n_{x} n_{x}^{\prime}}^{\pi_{x}}$ given by an expression like (2.7), where $p$ is replaced by $\pi_{x}$ and $n$ by $n_{x}$. We have $F_{\alpha \alpha^{\prime}}^{p}=F_{\alpha^{\prime} \alpha}^{p}$ which implies that $J_{+}(t)$ of Eq. (3.6) is in fact real. This quantity has been expressed in terms of $d_{n \alpha}^{\pi_{x} p}(L)$ only due to the relations (2.13).

In Figs. 3(a) and (b) the dotted line shows $J_{+}(t)$ obtained at $w=L / 2$ for $A=32$ and 80 particle systems, respectively. The result has been given over a full period which can be easily calculated. The one-sided current $J_{+}(t)$ returns to its initial zero value whenever

$$
e^{(i / \hbar)\left(E_{\alpha}^{p}-E_{\alpha^{\prime}}^{p}\right) t}=1 \text { for all } \alpha, \alpha^{\prime} .
$$

For the first two modes $E_{1}^{+}\left(n_{x}=n_{y}=n_{z}=1\right)$ and $E_{3}^{+}$ $\left(n_{x}=1, n_{y}=1, n_{z}=3\right)$ of the same parity the Eq. (3.9) is satisfied if

$$
T=\frac{2 L^{2} m}{\pi \hbar}
$$

Relation (3.10) gives a period of $J_{+}(t)$ because any other difference $E_{\alpha}^{p}-E_{\alpha^{\prime}}^{p}$ is a multiple of $E_{1}^{+}-E_{3}^{+}$when $w=L / 2$, and this makes condition (3.9) fulfilled for any $\alpha$ whenever $t=T$. Relation (3.10) gives $T=2.09 \times 10^{-22} \mathrm{~s}$ for $A=32$ and $T=3.85 \times 10^{-22}$ s for $A=80$.

Contrary to the periodical behavior at the fully open window Eqs. (3.9) have no unique solution at $w \neq L / 2$ because $E_{\alpha}^{p}$ are no more simply related and the current becomes aperiodical. This is illustrated in Figs. 4 and 5 for the $A=32$ system at $w=1.6$ and $1 \mathrm{fm}$. One can see that at $w=1.6 \mathrm{fm}$ the deviation from periodicity is still small, but at $w=1 \mathrm{fm}$ the periodicity has been entirely lost.

An interesting quantity is the time average $\bar{J}_{+}$of the current. Its analytic expression is

$$
\bar{J}_{+}=\frac{\hbar}{m} \frac{1}{L^{2}} \sum_{n(o c c)} \sum_{\alpha}\left[d_{n \alpha}^{\pi_{x}^{p}}(L)\right]^{2} F_{\alpha \alpha}^{p}
$$



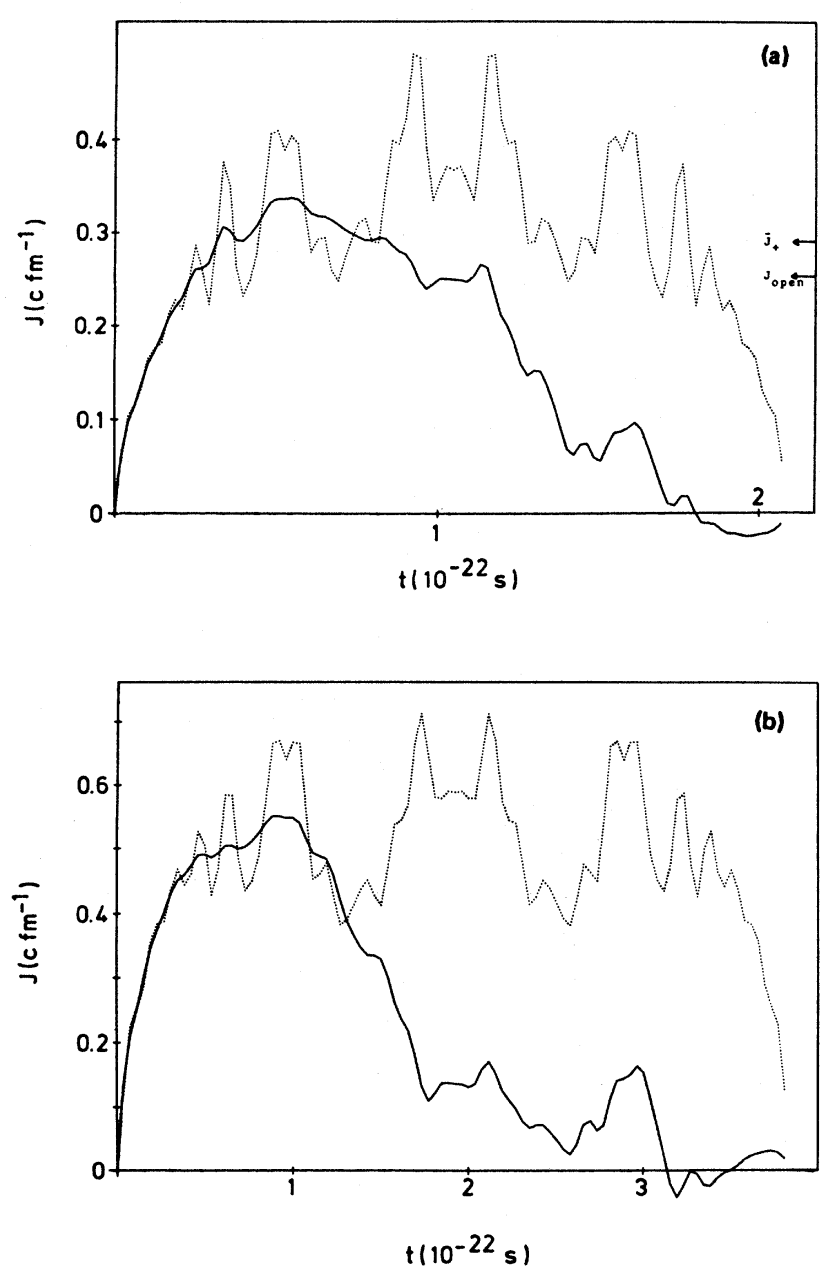

FIG. 3. The one-sided current $J_{+}$(dotted line) and $J^{L}$ (full line) as a function of time at $w=L / 2$. The parameters of the model are $\lambda=0, N=15$. (a) $A=32$ particles, (b) $A=80$ particles. The arrows indicate $\bar{J}_{+}$of Eq. (3.11) and $J_{\text {open }}=j_{\text {open }} \times 2 w L$ with $j_{\text {open }}$ from (3.12).

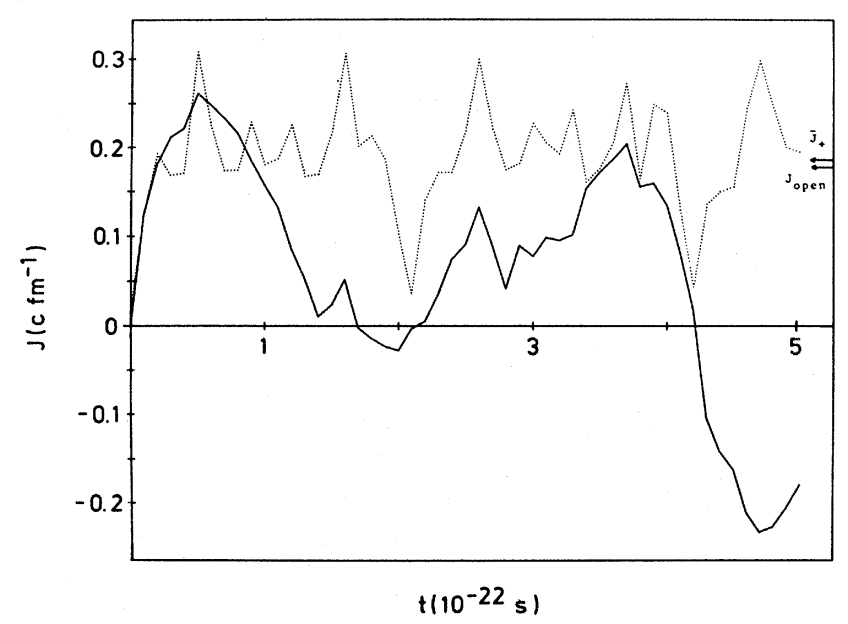

FIG. 4. Same as Fig. 3(a) but for $w=1.6 \mathrm{fm}, \lambda=10^{4} \mathrm{MeV} \mathrm{fm}$, $N=19$.

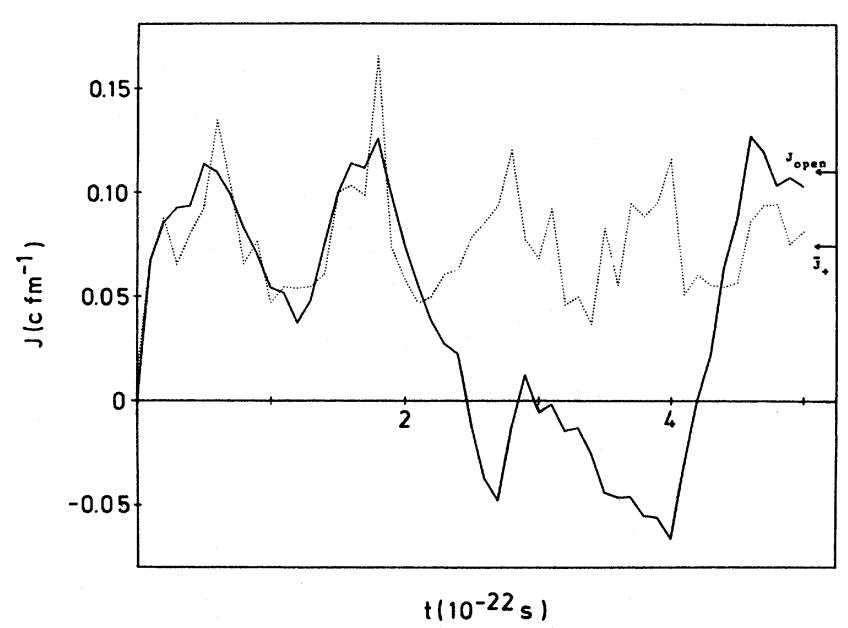

FIG. 5. Same as Fig. 3(a) but for $w=1 \mathrm{fm}, \lambda=10^{4} \mathrm{MeV} \mathrm{fm}$, $N=19$.

As an example, values of $\bar{J}_{+}$are given in Table II for $w=1$ and $1.6 \mathrm{fm}$ and $L / 2$ for the $A=32$ particle system. In Table II we also reproduce the values of the static onesided current obtained in Ref. 9 for the same values of $w$, $\lambda$, and $N$. It seems that there is a good agreement between the two results, the static value being somewhat smaller in all cases. The difference between the two results can be explained by the rearrangement in the eigenvector space of dimension $N$ through definition (2.9) when each component $\phi_{\alpha}^{p}$ has a weight $\left|d_{n \alpha}^{\pi_{x} p}\right|^{2}<1$, while in the static calculations the weight is either 1 or 0 .

For completeness we also reproduce the purely geometrical current obtained by multiplying the window area with the static one-sided flux $j_{\text {open }}$ obtained at maximum opening

$$
j_{\text {open }}=\frac{\hbar}{m} \frac{1}{L^{4}} \sum_{n_{z}(\text { occ })} n_{z} \operatorname{Si}\left(\pi n_{z}\right) .
$$

We note that a given $n_{z}$ can correspond to several sets $n_{x}, n_{y}$ and its multiplicity must be taken into account in (3.12).

\section{B. Definition II}

Now we consider one box full with $A / 2$ particles and the other empty at $t=0$. Let us choose the full left box. The window opens at $t>0$ and the particles flow into the other box. The current $J^{L}(t)$ is obtained as in Eq. (3.2), but from the flux $\left.j^{L}(\vec{r}, t)\right|_{z=0}$ given by the contribution of

TABLE II. The one-sided current for the $A=32$ particle system at three different window sizes $w ; \bar{J}_{+}$[Eq. (3.11)], $J_{\text {static }}$ (Ref. 9), $J_{\text {open }}=j_{\text {open }} \times 2 w L$ with $j_{\text {open }}$ from Eq. (3.12).

\begin{tabular}{lccc}
\hline \hline $\begin{array}{c}w \\
(\mathrm{fm})\end{array}$ & $\begin{array}{c}\bar{J}_{+} \\
\left(c \mathrm{fm}^{-1}\right)\end{array}$ & $\begin{array}{c}J_{\text {static }} \\
\left(c \mathrm{fm}^{-1}\right)\end{array}$ & $\begin{array}{c}J_{\text {open }} \\
\left(c \mathrm{fm}^{-1}\right)\end{array}$ \\
\hline 1 & 0.075 & 0.063 & 0.112 \\
1.6 & 0.187 & 0.162 & 0.179 \\
$L / 2$ & 0.288 & 0.254 & 0.254 \\
\hline \hline
\end{tabular}


all initially occupied states of the left box only

$$
\left.j^{L}(\overrightarrow{\mathrm{r}}, t)\right|_{z=0}=\left.4 \sum_{n \text { (occ) }} j_{n}^{L}(\overrightarrow{\mathrm{r}}, t)\right|_{z=0},
$$

where for $j_{n}^{L}$ we use the quantum-mechanical definition

$$
\left.j_{n}^{L}(\overrightarrow{\mathrm{r}}, t)\right|_{z=0}=\frac{\hbar}{m} \operatorname{Im}\left[\psi_{n}^{L}(\overrightarrow{\mathrm{r}}, t)^{*} \frac{d}{d z} \psi_{n}^{L}(\overrightarrow{\mathrm{r}}, t)\right]_{z=0}
$$

Using expansion (2.9) and definitions (2.3) and (2.13), we obtain

$$
J^{L}(t)=\frac{\hbar}{m} \frac{\pi \sqrt{2}}{L^{2}} \sum_{n(\mathrm{occ})} \sum_{\alpha \neq n} n_{z} d_{n \alpha}^{+1}(L) \sin \frac{E_{\alpha}^{p}-E_{n}^{-p}}{\hbar} t \sum_{n^{\prime \prime}} c_{\alpha n^{\prime \prime}}^{p} B_{n_{x} n_{x}^{\prime \prime}}^{p}
$$

where $n_{z}$ is even and $n_{z}^{\prime \prime}$ odd. Although by definition $d_{n \alpha}^{+1}$ implies $\alpha \neq n$ we specify it in the summation in order to avoid any confusion here or below.

In Figs. 3-5 the result for $J^{L}(t)$ is represented by a full line. In all cases one can see that there is an interval starting at $t=0$ where $J_{+}$and $J^{L}$ are very similar. This interval increases as $w$ decreases. Its length depends on the moment when the reflection on the opposite wall starts to dominate. In a large time interval $J^{L}$ oscillates between positive and negative values showing the emptying and filling up of the left box, while $J_{+}$remains always positive. The region where $J_{+}$and $J^{L}$ are close to each other plays an important role. By construction $J^{L}$ has a one-sided nature before the reflection sets in, i.e., the particles leave the left box with $k_{z}>0$ and travel into the right box. The practical coincidence between $J_{+}$and $J^{L}$ shows that $J_{+}^{R}$, i.e., the contribution to $J_{+}$coming from the particles initially present in the right box is negligible or vice versa whenever such a contribution is negligible, the exclusion of the interval $\int_{-\infty}^{0} d k_{z}$ in the calculation of the flux, as it is done in Eq. (3.1), is entirely justified. These considerations are illustrated in Figs. 6 and 7 for the system with $A=32$ particles with a window of size $w=1 \mathrm{fm}$. In Fig. 6 we compare $J^{L}$ with $J_{+}^{L}$, i.e., the one-sided flux (3.2) obtained by filling the left box only with $A / 2$ particles at $t=0$. Comparing $J_{+}=J_{+}^{L}+J_{+}^{R}$ from Fig. 5 with $J_{+}^{L}$ from Fig. 6, we see that $J_{+}^{R}$ becomes appreciable only beyond $t>2 \times 10^{-22} \mathrm{~s}$, while $J_{+}^{L}$ remains dominant from $t=0$ up to $t \sim 2 \times 10^{-22} \mathrm{~s}$. In the Appendix we show that

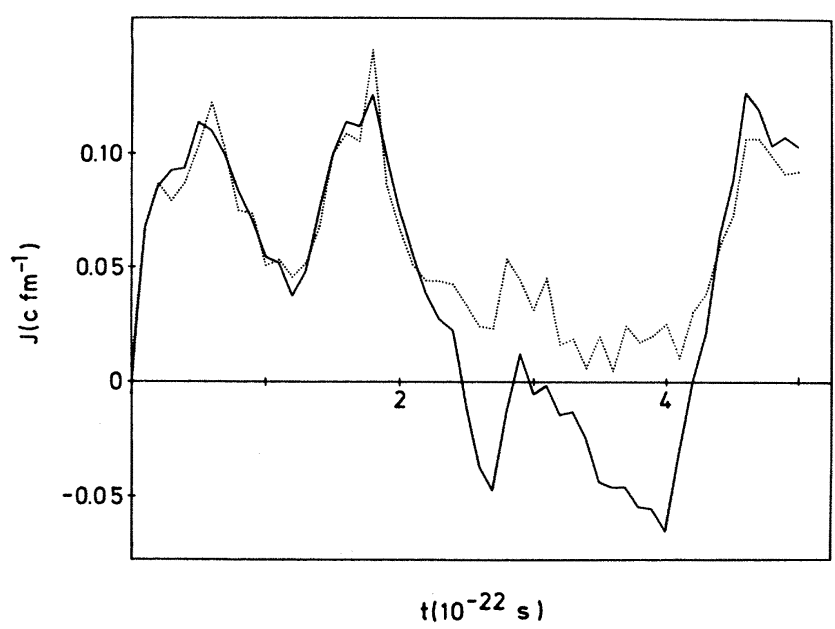

FIG. 6. The one-sided current for the full left box with $A / 2$ particles, empty right box: $J_{+}^{L}$ (dotted line) and $J^{L}$ (full line) as a function of time at $w=1 \mathrm{fm}$ and $A=32$ particles. The parameters are $\lambda=10^{4} \mathrm{MeV} \mathrm{fm}, N=19$.

$$
\begin{aligned}
& J_{+}^{L}=\frac{1}{2}\left(J_{+}+J^{L}\right), \\
& J_{+}^{R}=\frac{1}{2}\left(J_{+}-J^{L}\right),
\end{aligned}
$$

where $J_{+}$and $J^{L}$ are given by Eqs. (3.6) and (3.15), respectively. When the two boxes are equally filled with $A / 4$ particles $J^{L}$ becomes zero and only at this moment $J_{+}^{R}$ and $J_{+}^{L}$ become equal. In Fig. 7 we compare $J_{+}^{L}$ with a quantity called $J_{-}^{L}$ defined as the current (3.2), but obtained from

$$
j_{-}^{L}=\frac{\hbar}{m} \int_{k_{z}<0} d^{3} \overrightarrow{\mathrm{k}} k_{z} f^{L}(\overrightarrow{\mathrm{r}}, \overrightarrow{\mathrm{k}}, t),
$$

where the Wigner function $f^{L}$ is associated with the full left box and the empty right box. One can see that over the interval where $J_{+}^{R}$ is negligible, $J_{-}^{L}$ is also negligible as we have previously mentioned. In fact, through the use of some algebra like in the Appendix and the fact that the Wigner function is symmetric under the reflections $\overrightarrow{\mathrm{k}} \rightarrow-\overrightarrow{\mathrm{k}}$, one can show that $J_{+}^{R}$ and $J_{-}^{L}$ are equal but of opposite sign.

\section{THE WIDTH OF THE MASS DISTRIBUTION}

In the preceding section we have shown results for the current which gives a measure of the nucleon exchange between nuclei. In heavy ions another quantity of interest is the width of the mass or charge distribution. While the current is related to the one-body density via the continuity equation, the width of the mass distribution is expressed in terms of the two-body density matrix. Within the present model the variance $\sigma^{2}$ of the particle number

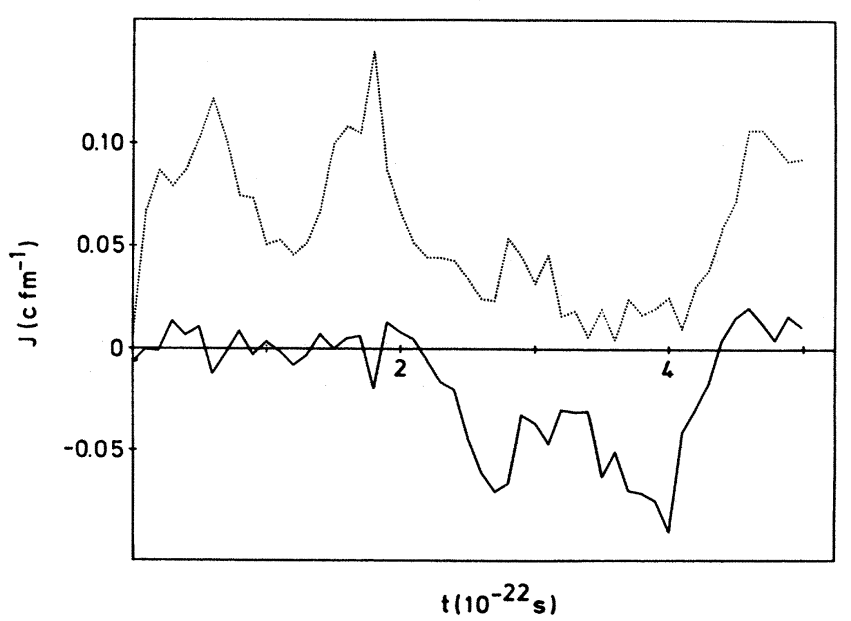

FIG. 7. Same as Fig. 6 but full line gives $J_{-}^{L}$ defined according to (3.2) and (3.17). 
in one box can be easily calculated.

We define the particle number operator in one box like in Refs. 7 and 12. If we arbitrarily choose the left box this operator is

$$
N_{L}=\sum_{i=1}^{A} n_{L}(i)=\sum_{i=1}^{A}\left[1-\theta\left(z_{i}\right)\right]
$$

with $A$ the total number of particles and $\theta\left(z_{i}\right)$ the step function

$$
\theta\left(z_{i}\right)=\left\{\begin{array}{l}
1 \text { for } z_{i}>0 \\
0 \text { for } z_{i}<0
\end{array}\right.
$$

The variance is

$$
\begin{array}{ll}
\sigma_{L}^{2}=\left\langle N_{L}^{2}\right\rangle-\left\langle N_{L}\right\rangle^{2}, & \text { (4.3) } \\
\left\langle o\left|n_{L}\right| u\right\rangle=\frac{1}{2} & \sum_{\alpha \neq o, u} d_{o \alpha}^{+1}(L) d_{u \alpha}^{+1}(L)\left[1 \pm\left(e^{(i / \hbar)\left(E_{\alpha}^{p}-E_{u}^{-p}\right) t}+e^{-(i / \hbar)\left(E_{\alpha}^{p}-E_{o}^{-p}\right) t}\right)\right] \text { for } o \neq u,
\end{array}
$$

where the expectation value \langle\rangle is taken with respect to the ground state. In the present model this state is a Slater determinant. Hence,

$$
\sigma_{L}^{2}=\sum_{o, u}\left|\left\langle o\left|n_{L}\right| u\right\rangle\right|^{2},
$$

where $o$ and $u$ represent occupied and unoccupied states, respectively. We discuss the case of two equally filled boxes. Then the states $o, u$ can either be chosen to have a definite parity or be represented by wave functions initially localized in the left or right box as given by Eq. (2.9). In the first version the procedure would be close to a twocenter shell model description. ${ }^{12}$ In the second version the parities are mixed in the wave functions, but for continuity we maintain this basis such as it has been defined in Sec. II. Then the matrix element $\left\langle o\left|n_{L}\right| u\right\rangle$ is given by

where the plus (minus) sign is related to the case where both states $|o\rangle$ and $|u\rangle$ are initially localized in the left (right) box. If $|o\rangle$ and $|u\rangle$ are initially in different boxes the expression is

$$
\left\langle o\left|n_{L}\right| u\right\rangle=\frac{1}{2} \sum_{\alpha \neq o, u} d_{o \alpha}^{+1}(L) d_{u \alpha}^{+1}(L)\left[-1 \pm\left(e^{(i / \hbar)\left(E_{\alpha}^{p}-E_{u}^{-p}\right) t}-e^{-(i / \hbar)\left(E_{\alpha}^{p}-E_{o}^{-p}\right) t}\right)\right] \text { for } o \neq u,
$$

with the $+(-)$ sign for $|o\rangle$ in the left (right) box, respectively. If the number of states $\alpha$ is large enough the orthogonality between $|u\rangle$ and $|o\rangle$ is well satisfied, which implies

$$
\sum_{\alpha \neq 0, u} d_{o \alpha}^{+1}(L) d_{u \alpha}^{+1}(L) \simeq 0 \text { for } o \neq u
$$

Using (4.7) and adding up the four contributions given by (4.5) and (4.6), we obtain

$$
\sigma_{L}^{2}=2 \sum_{o \neq u}\left|\sum_{\alpha \neq o, u} d_{o \alpha}^{+1}(L) d_{u \alpha}^{+1}(L) e^{i / \hbar E_{\alpha}^{p} t}\right|^{2}
$$

The calculated value of $\sigma_{L}^{2}$ is shown in Figs. 8(a) and (b) for the systems $A=32$ and 80 , respectively, at $w=L / 2$. One can notice a periodical structure again, like for the current $J_{+}$. Each time the condition

$$
e^{(i / \hbar) E_{\alpha}^{p} t}=1 \text { for all } \alpha
$$

is fulfilled, $\sigma_{L}^{2}$ goes to zero. This takes place at the same period $T$ given by (3.10), which can be easily seen if we use instead of (4.9) the equation

$$
e^{(i / \hbar)\left(E_{\alpha}^{p}-E_{1}^{p}\right) t}=1,
$$

also consistent with (4.8). As already mentioned the period $T$ for $A=80$ is about twice as large than that for $A=32$. If we average over a period, the formula (4.8) gives

$$
\overline{\sigma_{L}^{2}}=2 \sum_{o \neq u} \sum_{\alpha \neq o, u}\left[d_{o \alpha}^{+1}(L) d_{u \alpha}^{+1}(L)\right]^{2}
$$

because the coefficients $d_{n \alpha}^{\pi_{x} p}$ are real. This quantity should be compared to the classical value ${ }^{7,13}$ of $\sigma_{L}^{2}$ which at large $t$ tends towards $A / 4$, where $A$ is the total number of particles. The ratio between $\overline{\sigma_{L}^{2}}$ and the asymptotic classical value is $2 / 8$ for $A=32$ and $4.88 / 20$ for $A=80$,
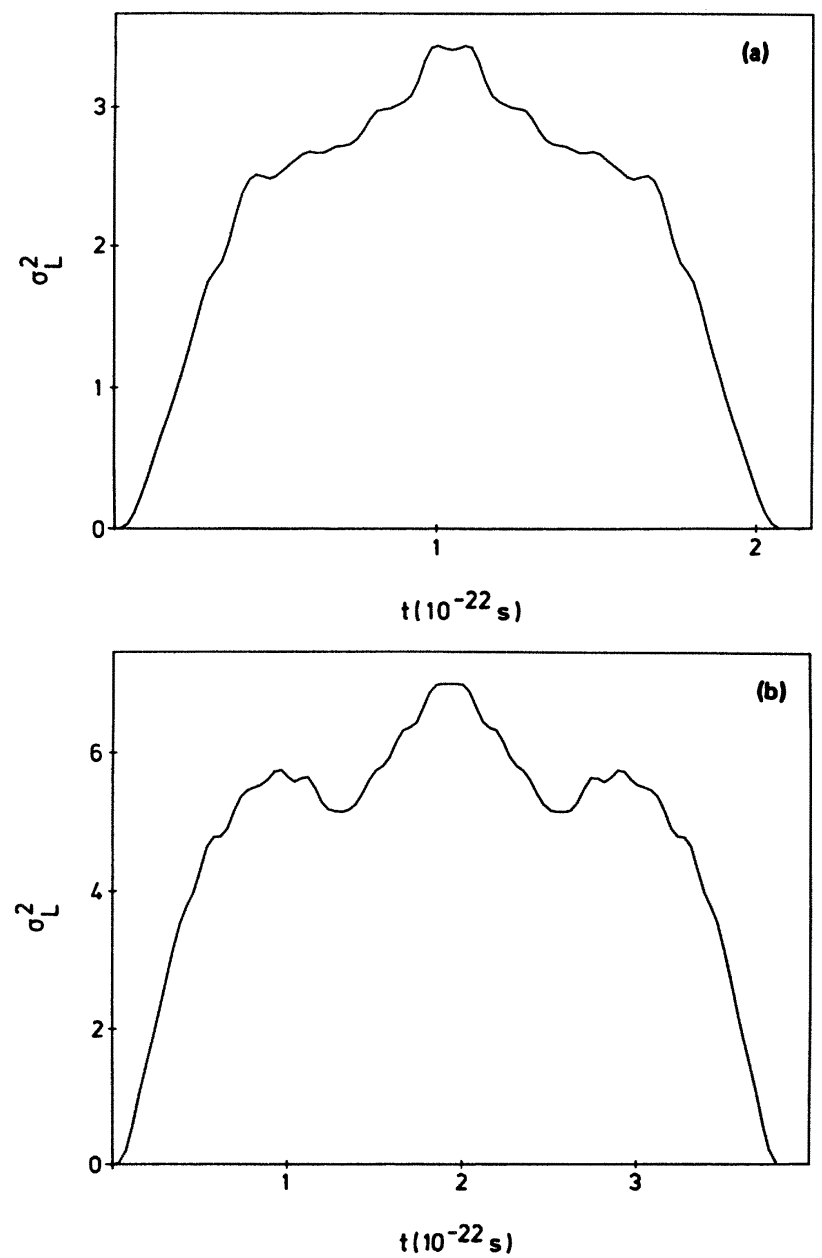

FIG. 8. The width of the mass distribution $\sigma_{L}^{2}$ as a function of time at $w=L / 2$ and $N=15$. (a) $A=32$ particles; (b) $A=80$ particles. 
i.e., it has the same order of magnitude as that found in Ref. 7 from a one-dimensional model. This confirms the conclusion given in Ref. 7 that when the particles both at the left and right are forced to move in the same potential the mass dispersion is very small, as in time-dependent Hartree-Fock calculations. It would be interesting to extend the present study to the case where the two boxes have different sizes in order to see whether $\sigma^{2}$ increases in such a case.

\section{CONCLUSIONS}

We have formulated a simple three-dimensional quantum-mechanical independent particle model in order to get more insight into the quantities describing the exchange of nucleons between heavy ions. In this model the colliding nuclei are represented by two adjacent cubic boxes communicating through a rectangular window. The window suddenly opens with a given size and we calculate the time evolution of the one-sided current and of the width of the mass distribution at constant opening.

We have used two different definitions to calculate the one-sided flux of the window formula. One is the currently used definition introduced as an extension of the classical definition and based on the Wigner distribution function obtained from the single particle wave functions of the communicating system. The other is the quantum- mechanical definition applied in the case where one box is filled and the other box is empty. The calculations show that both definitions give similar results in the time interval before the reflection on the opposite wall sets in. We consider that such similarities bring support in using the extended classical definition where the integration over positive momenta only [see Eq. (3.1)] is not a priori justified in quantum mechanics.

The calculated width of the mass distribution $\sigma_{L}^{2}$ has a periodical structure, like $J_{+}$, due to the finite size of the system. The ratio between the time average of $\sigma_{L}^{2}$ and its asymptotic classical value has the same order of magnitude as that obtained by Buck and Feldmeier ${ }^{7}$ in a onedimensional system. Our calculations therefore support the conclusion of Ref. 7 according to which the smallness of $\sigma_{L}^{2}$ is the combined effect of the Pauli principle and the use of an identical mean potential for both nuclei.

One of us (C.G.) acknowledges financial support from the Institute Interuniversitaire des Sciences Nucléaires.

\section{APPENDIX}

By using definition (2.9) and relations (2.13) one can write the density matrix associated with a state $n$ initially occupied in the left box as

$$
\begin{aligned}
\rho_{n}^{L}\left(\overrightarrow{\mathrm{r}}, \overrightarrow{\mathrm{r}}^{\prime}, t\right)= & \sum_{\alpha} d_{n \alpha}^{\pi_{x} p}(L) d_{n \alpha^{\prime}}^{\pi_{x}^{p}}(L) e^{(i / \hbar)\left(E_{\alpha}^{p}-E_{\alpha^{\prime}}^{p}\right) t} \phi_{\alpha}^{p}(\overrightarrow{\mathrm{r}}) \phi_{\alpha^{\prime}}^{p}\left(\overrightarrow{\mathrm{r}}^{\prime}\right) \\
& +\frac{\sqrt{2}}{2} \sum_{\alpha \neq n} d_{n \alpha}^{+1}(L)\left[e^{\left.(i / \hbar) E_{\alpha}^{p}-E_{n}^{-p}\right) t} \phi_{\alpha}^{p}(\overrightarrow{\mathrm{r}}) \phi_{n}^{-p}\left(\overrightarrow{\mathrm{r}}^{\prime}\right)+e^{-(i / \hbar)\left(E_{\alpha}^{p}-E_{n}^{-p}\right) t} \phi_{n}^{-p}(\overrightarrow{\mathrm{r}}) \phi_{n}^{p}\left(\overrightarrow{\mathrm{r}}^{\prime}\right)\right]
\end{aligned}
$$

i.e., as a sum of two distinct terms: the first term contains products of states $\phi_{\alpha}^{p}$ and $\phi_{\alpha^{\prime}}^{p}$ of the same parity, while in the second term one wave function carries the parity $-p$ of the initially occupied state and the other has the opposite parity $p$.

By using the same relations one finds that the density matrix $\rho_{n}^{R}$ associated with a state $n$ initially occupied in the right box is given by the difference between the same two terms.

In calculating $J_{+, n}^{L}$ or $J_{+, n}^{R}$ according to (3.5), (3.1), and (3.2) one can treat the two terms separately. Making the summation over all occupied states, each four times degenerate, one obtains

$$
\begin{aligned}
& J_{+}^{L}=4 \sum_{n \text { (occ) }} J_{+, n}^{L}=\frac{1}{2}\left(J_{+}+J^{L}\right), \\
& J_{+}^{R}=4 \sum_{n \text { (occ) }} J_{+, n}^{R}=\frac{1}{2}\left(J_{+}-J^{L}\right) .
\end{aligned}
$$

In (A2) $J_{+}$is given by (3.6) and originates from the first term in (A1) and $J^{L}$ is given by (3.15) and originates from the second term of (A1). Similar considerations are valid for (A3) derived from $\rho_{n}^{R}$.
${ }^{1}$ W. Nörenberg and H. A. Weidenmüller, Introduction to the Theory of Heavy-Ion Collisions, Lecture Notes in Physics, edited by J. Ehlers, K. Hepp, H. A. Weidenmüller, and J. Zittartz (Springer, Berlin, 1976).

2J. Blocki, Y. Boneh, J. R. Nix, J. Randrup, M. Robel, A. J. Sierk, and W. J. Swiatecki, Ann. Phys. (N.Y.) 113, 330 (1978).

${ }^{3}$ W. J. Swiatecki, J. Phys. 33, C5-45 (1972).

${ }^{4}$ M. Prakash, S. Shlomo, B. S. Nilsson, J. P. Bondorf, and F. E. Serr, Phys. Rev. Lett. 47, 898 (1981); Nucl. Phys. $\underline{\text { A385, }} 483$ (1982).

${ }^{5} \mathrm{~K}$. Goeke, F. Grümmer and P.-G. Reinhard, Ann. Phys. (N.Y.) (in press); (private communication).

${ }^{6} \mathrm{H}$. Feldmeier, Lawrence Berkeley Laboratory Report LBL-
10688, UC-34C, CONF-800329, 1980.

${ }^{7}$ P. Buck and H. Feldmeier, Nucl. Phys. A394, 334 (1983).

${ }^{8}$ C. Gnucci and Fl. Stancu, Phys. Rev. C (to be published).

${ }^{9}$ C. Gnucci, Fl. Stancu, and L. Wilets, Phys. Lett. B127, 10 (1983).

${ }^{10}$ C. Gnucci, Ph.D. thesis, University of Liège, 1983 (unpublished).

${ }^{11}$ M. Abramowitz and I. A. Stegun, Handbook of Mathematical Functions (Dover, New York, 1968), p. 232.

${ }^{12}$ C. Schöne, M. Diebel, and U. Mosel, Z. Phys. A 294, 351 (1980).

${ }^{13}$ F. Beck, M. Dworzecka, and H. Feldmeier, Z. Phys. A $\underline{289}$, 113 (1978). 